\title{
Quand la médecine nous prend par les sentiments: Le magnétiseur amoureux de Charles de Villers*
}

Dolores Martín Moruno

\section{Summary}

This article analyses the representation of affective phenomena brought into play in Charles de Villers' Le magnétiseur amoureux (1787) as it helps to better understand the historical transition from the Galenic conception of the passions of the soul to the cerebral interpretation of emotions. While feelings became the condition of possibility in the occurrence of the therapy, passion is identified as the cause of the young woman's illness, Caroline, who according to the interpretation proposed in this article, suffers from what was identified in the eighteenth century medical tradition as «love melancholy» or «hysteric affection», which were both pathologies that alluded to vapors in order to explain their symptoms. The analysis of the logic of feeling running across Villers' novel impels us to interpret the magnetic fluid in terms of the sympathy created between the two main characters. The ambivalence expressed by Villers between the meaning of «love sentiment» and that of «love passion» allows us to finally understand the somnambulist therapy as erotic knowledge that implies a reflection on love codes in late eighteenth century France.

Keywords: History of emotions, feelings, love's passion, artificial somnambulism, vapors, hysteric affection, Charles de Villers

* Cet article est inspiré de la présentation d'un travail de recherche intitulé «La constitution d'un discours magnétique sur les passions à la fin du $18^{\text {ème }}$ siècle» dans le cadre du colloque Le Mesmérisme en contexte organisé par Bruno Belhoste et Nicole Edelman le 19 juin 2009 à l'auditorium du Château de Versailles. Je remercie Elisa Andretta, Sophie Milquet et Luis Montiel pour leurs suggestions ainsi que les critiques des expertises anonymes, qui ont été infiniment précieuses pour l'élaboration de cet article.

Dolores Martín Moruno, iEH2, Faculté de Médecine, Centre Médical Universitaire, CH-1211 Genève 4 (dolores.martinmoruno@unige.ch) 


\section{Résumé}

Cet article analyse la représentation des phénomènes affectifs mis en jeu dans Le magnétiseur amoureux (1787) de Charles de Villers dans le but de mieux comprendre la transition historique entre la conception galénique des passions de l'âme et l'interprétation cérébrale des émotions. Alors que les sentiments deviennent la condition de possibilité pour que la thérapie ait lieu, la passion est identifiée comme la cause de la maladie d'une jeune femme, Caroline, qui d'après l'interprétation proposée dans cet article souffre de ce qui était compris par la tradition médicale du XVIII ${ }^{\mathrm{e}}$ siècle comme «mélancolie érotique» ou «affection hystérique», des pathologies qui évoquaient des vapeurs pour expliquer leurs symptômes. L'analyse de la logique du sentiment traversant le roman de Villers suggère d'interpréter le fluide magnétique comme une sympathie entretenue entre les deux personnages principaux. L'ambivalence exprimée par Villers entre la signification du sentiment et celle de la passion amoureuse permet enfin de comprendre la thérapie somnambulique comme une connaissance érotique qui implique une réflexion sur les codes amoureux de la France de la fin du XVIII ${ }^{\mathrm{e}}$ siècle.

Mots-clés: Histoire de l'affectivité, sentiments, passion amoureuse, somnambulisme artificiel, affection hystérique, Charles de Villers

\section{Les sentiments dans le somnambulisme artificiel de Charles de Villers}

Cet article analyse la représentation des sentiments et passions dans Le magnétiseur amoureux (1787) de Charles de Villers, un roman dont le fil conducteur est la description d'une cure magnétique qui a pour but de guérir une jeune femme de dix-huit ans «d'une santé chancelante». ${ }^{1}$ Nous verrons que les sentiments introduits par Villers dans ce roman viennent remplacer le fluide physique que Franz Anton Mesmer avait nommé «magnétisme animal» ${ }^{2}$ et définissent ainsi la thérapie somnambulique par l'intermédiaire de la notion de «sympathie», ce que nous pourrions aujourd'hui qualifier de rapport de nature émotionnelle. ${ }^{3}$

L'intérêt historique de ce roman réside dans le fait qu'il constitue une source textuelle où l'on peut «ausculter l'émotion qui se dit ou qui se montre

1 Villers 2006, 85.

2 Mesmer 1779, 6.

3 L'élimination du fluide chez Villers a été discuté par Azouvi 1996, 12 et Méheust 1998, 135.

Sur la définition de «sympathie», voir le Dictionnaire de l'Académie française 1798, 621. 
par des gestes visibles ou descriptibles» et dont l'étude peut contribuer à éclaircir notre manière contemporaine de comprendre la vie affective par rapport à la conception de la sensibilité en vigueur à la fin du XVIII ${ }^{e}$ siècle. ${ }^{4}$ Du point de vue de ce que certains historiens anglophones ont appelé «l'histoire des émotions» et que d'autres historiens francophones ont revendiqué comme une «histoire de l'affectivité», 5 Le magnétiseur amoureux propose une compréhension extrêmement intéressante du rôle que joue l'affectivité dans la pratique médicale ainsi que dans la maladie, car celle-ci n'est pas exclusivement considérée comme la source d'une «douleur physique» mais aussi comme une «peine morale» qui montre «l'union intime de l'âme et du corps». ${ }^{6} \mathrm{~A}$ la différence d'autres interprétations de ce texte littéraire, et notamment celle proposée par le philosophe François Azouvi, cet article vise à étudier la représentation des phénomènes affectifs mis en jeu dans ce roman car elle peut nous aider à mieux comprendre cette période de transition entre la tradition galénique des passions de l'âme et l'interprétation de la vie affective au regard du concept scientifique d'émotion, une construction théorique introduite par la psychologie au cours du XIX ${ }^{\mathrm{e}}$ siècle. ${ }^{7}$

Mais l'intérêt principal de l'analyse de ce roman est aussi comme l'obstacle principal pour sa compréhension. En effet, Villiers ne s'exprime pas avec le terme actuel «d'émotion», mais utilise des mots tels que «sentiments», «passions», «sympathie» et «affections». Cette variation du lexique n'implique pas seulement une distance linguistique, mais aussi des conceptions historiques distinctes du corps qui ne définissent pas le siège organique de la vie affective dans le cerveau comme nous l'acceptons aujourd'hui. ${ }^{8}$ A la fin du XVIII ${ }^{\mathrm{e}}$ siècle, le sentiment était une catégorie assez ample qui désignait la «perception que l'âme a des objets par le moyen des organes des

4 Boquet et Nagi 2008, 39.

5 L'expression anglaise «history of emotions» a été introduite par Peter Stearns, William Reddy et Barbara Rosenwein, considérés les fondateurs de ce programme de recherche. A ce sujet, voir Pampler 2010, 237. Par contre, des historiens francophones comme Boquet et Nagi 2011, 11 ont proposé l'appellation alternative «d'histoire de l'affectivité» pour se référer à «l'étude des dispositions affectives et des traits du caractère, aux sentiments durables et aux émotions qui, en français désignent clairement un mouvement psychique bref, le plus souvent reflété par le corps».

6 Villers 2006, 119.

7 Sur l'apparition scientifique du terme «émotion» dans la littérature anglophone, il faut consulter Dixon 2003, 23. Dans la littérature francophone, Ribot 2007, 189, divise les sentiments en trois groupes: «les états affectifs communs et d'intensité moyenne, les émotions et les passions». Il comprend le terme «d'émotion» par opposition à celui de «passion». Ribot 1998, 7374: «J'entends par émotion un choc brusque, souvent violent, intense, avec augmentation ou arrêt des mouvements [...] J'entends par passion une émotion devenue fixe [...]».

8 L'importance d'une étude lexicale préalable afin de développer «une histoire des sentiments» a été soulignée par Starobinski 2013, 259. Voir aussi Vidal 2009, 5 et Bound-Alberti 2010, 6. 
sens», mais qui «se disait encore des affections, des passions et de tous les mouvements de l'âme». ${ }^{9}$ Cette notion s'inscrivait généralement dans le cadre d'une physiologie du système nerveux ou les fibres et les nerfs étaient considérés comme les fondements du corps. ${ }^{10}$

Comme nous allons le voir, les sentiments occupent une place privilégiée dans la thérapie décrite par Villers car ils ne sont pas seulement «la manière dont les impressions des objets extérieurs se transmettent à l'âme», mais aussi l'affection «de l'âme compatissante» du médecin envers la souffrance du malade; affection qui est d'ailleurs interprétée dans le roman dans les termes du sentiment amoureux. ${ }^{11}$ Avant d'examiner la logique du sentiment traversant le récit du Magnétiseur amoureux, il nous faut d'abord introduire la personnalité de Charles de Villers et expliquer la nature de sa contribution par rapport à la tradition du magnétisme animal de Mesmer et du somnambulisme artificiel d'Armand-Marie-Jacques de Chastenet, plus connu sous le nom de Marquis de Puységur (1751-1825).

Charles François Dominique de Villers (1765-1815) était originaire d'une petite ville de Boulay, Bolchen, dont la proximité avec l'Allemagne annonce déjà - selon ses biographes - son futur rôle de médiateur entre la culture française et germanique à côté d'autres personnalités de renommée comme Germaine de Staël. ${ }^{12}$ Après avoir suivi une formation au Collège des Bénédictins à Metz, il s'enrôle dans le Régiment d'Artillerie en 1783 où, deux ans plus tard, il allait rencontrer le marquis de Puységur, alors colonel d'artillerie commandant du régime royal de Strasbourg.

C'est dans cette ville que Puységur fonde La Société Harmonique du Régiment de Metz, l'endroit où Villers se familiarise avec la doctrine du magnétisme animal et plus particulièrement, avec la découverte que Puységur fait le 4 mai 1784 en magnétisant un malade dont le nom est Victor et qui, au lieu d'entrer dans un état de transe convulsive, semble s'endormir. Malgré son apparence, le malade est capable de répondre aux questions posées par Puységur, montrant ainsi un sens de la lucidité et qui lui permet de diagnostiquer la cause de sa souffrance et de pronostiquer quand et comment il va guérir. C'est cet état de «sommeil ambuliste» qu'il appelle «somnambulisme magnétique», ${ }^{13}$ une modification de la conscience qui

9 Dictionnaire de l'Académie française 1798, 558.

10 Voir Rousseau, G. 2004 et Wenger 2007.

11 Villers 2006, 118 et 99. Voir aussi, Dictionnaire de l'Académie française 1798, 27. L'affection est définie comme «amour, le sentiment qui fait qu'on aime, qu'on préfère quelque personne, qu'on se plaît à quelque chose, qu'on s'y porte avec ardeur».

12 Bégin 1832, 410 et Wittmer 1908, ii.

13 Puységur 1786, 89 et Puységur 1809,121. Voir aussi Peter 1999. 
deviendra une fascination dans la culture européenne du XIX ${ }^{\mathrm{e}}$ siècle inspirant des pratiques comme l'hypnose de James Braid. ${ }^{14}$

Cette thérapie, que Puységur décrivait comme «l'art de guérir par des paroles jointes $»^{15}$ est le point de départ du Magnétiseur amoureux de Villers, un ouvrage qui paraît trois ans après que soit rendu public le rapport des commissaires sur la valeur scientifique du magnétisme; rapport qui refusait l'existence du fluide et attribue ses effets, entre autres, à l'imagination des patients. ${ }^{16}$ Comme l'a indiqué François Azouvi, qui a tiré de l'oubli ce roman en 1978 grâce à la réédition de l'unique exemplaire alors connu, ${ }^{17}$ c'est dans le contexte de la polémique opposant partisans et détracteurs du magnétisme animal qu'il faut situer la contribution de Villers. Cette polémique fut si virulente que dès la parution anonyme du Magnétiseur amoureux, le livre allait être interdit par la police royale de Louis XVI qui détruisit la plupart des exemplaires imprimés. ${ }^{18}$ Le roman allait ainsi passer presque inaperçu jusqu'en 1824, quand le marquis de Puységur décida de le rééditer en modifiant profondément le texte, une fois Villers décédé. ${ }^{19}$

L'une des hypothèses les plus probables pour expliquer l'interdiction du livre de Villers est qu'il a dû éveiller les soupçons des autorités sur son possible contenu érotique; soupçons déjà renforcés par le titre du roman mettant en avant les connotations sexuelles des rapports établis lors de la thérapie entre le magnétiseur, qui était toujours un homme, et la patiente, qui était dans la plupart des cas une femme souffrant d'une maladie nerveuse. ${ }^{20}$ Cette

14 Afin de comprendre les rapports entre l'hypnose de James Braid et le somnambulisme, il convient de consulter la revue du magnétiseur genevois Charles Lafontaine 1860, 8 où cet auteur explique comment Braid a développé sa théorie après avoir assisté à une des performances magnétiques de ce dernier à Manchester.

15 Puységur 1809, 64. Pour une interprétation plus récente, voir Peter 2009, 32.

16 Bailly 1784, 37-8. Trois autres rapports sur le magnétisme animal ont étés rédigés en 1784: le «Rapport secret adressé au roi, sur le prétendu magnétisme animal» signé par Franklin et rédigé par Bailly et deux autres appartenant à la Société Royale de médicine, le «Rapport des commissaires de l'Académie Royale de Médicine nommés par le roi pour faire l'examen du magnétisme animal» et le «Rapport particulier de l'un des commissaires de la Société royale de médecine sur le magnétisme animal».

17 Villers 1787. Il faut ajouter aujourd'hui à l'unique exemplaire que François Azouvi connaissait et qui est conservé à la bibliothèque de Besançon, deux autres exemplaires hébergés par la Bibliothèque de Reims (Coté Rés. Diancourt P.1090) et par le Museum d'histoire naturelle de Paris Fonds anciens (Coté CH 147). Il existe également une copie microfilmée d'un exemplaire éliminé récemment pour cause de vétusté à la Bibliothèque de Genève (Ve 1368).

18 Wittmer 1908, 6et Azouvi 2006, 25.

19 Puységur 1824.

20 Dictionnaire de l'Académie française 1798, 569. La définition du terme «sexuelle» à la fin du XVIII ${ }^{\mathrm{e}}$ siècle se référait aux «différences caractérisées par les parties sexuelles», c'est-à-dire à ce que nous comprenons aujourd'hui comme «sexe» et pas comme «sexualité». Ces différences sexuelles, et en particulier l'utérus, expliquaient l'apparition de certaines maladies nerveuses comme la mélancolie et les vapeurs chez la femme qui étaient d'ailleurs les souffrances par excellence traitées par les magnétiseurs. A ce propos, consulter le rapport signé par Bailly 1784, 35-7. 
explication prend force lorsqu'on s'aperçoit que dans un des rapports secrets issus des commissions de 1784 , les commissaires signalent que «le traitement magnétique ne peut être que dangereux pour les mœurs» car «on excite des émotions agréables et chères [...] que l'on cherche à retrouver parce qu'elles ont un charme naturel [...] mais moralement elles n'en sont pas moins condamnables». ${ }^{21}$

Avec le choix d'un titre comme Le magnétiseur amoureux, Villers semblait vouloir répondre aux critiques des commissaires reconnaissant l'érotisme implicite dans le magnétisme comme un aspect fondamental de la thérapie, c'està-dire comme une force qui, utilisée dans «les bornes du respect», peut avoir des vertus curatives. ${ }^{22}$ Ainsi, cette défense des rapports amoureux chez Villers s'écarte explicitement de la tradition libertine représentée par Choderlos de Laclos ou Donatien Alphonse François de Sade à laquelle il reproche ouvertement de faire «l'apologie du vice» dans une lettre publiée en 1797.23

Comme nous allons le voir dans cette étude critique du Magnétiseur amoureux, ce roman permet de repenser le magnétisme animal en tant que médecine qui implique la participation des sentiments, et notamment de l'amour, comme une force intrinsèque aux rapports magnétiques établis lors de la thérapie. Ces rapports entre le magnétiseur et la patiente se traduisent dans le roman par son ressort principal: l'affection qui germe entre Valcourt et Caroline et qui symbolise l'attraction entre les deux pôles d'un aimant. De cette façon,l'amour est conçu par analogie au magnétisme qui,loin d'être imaginé comme un fluide physique, apparaît comme le résultat d'un «transport de l'âme» ou une «identification», une sorte de communication établie grâce à la convergence de la volonté de l'opérateur qui vient s'ajouter à celle du malade. ${ }^{24}$

Contrairement aux sentiments, la passion amoureuse est comprise par Villers comme étroitement liée à l'expérience d'une souffrance. Cette dimension douloureuse, et même pathologique, de l'amour est renforcée dans le roman quand cette passion est identifiée comme la cause de la maladie qui menace la jeune Caroline, qui - d'après l'interprétation proposée dans la section suivante - souffrait de ce que la tradition médicale désignait comme maladie d'amour ou mélancolie érotique et qu'au cours du XVIII ${ }^{\mathrm{e}}$ siècle on associait à des pathologies comme l'hystérie, trouble résultant des effets de vapeurs provenant de l'utérus chez la femme. ${ }^{25}$

21 Bailly 1784, 514.

22 Villers 2006, 96. Voir aussi, Rausky 1977, 237.

23 Villers 1797, 23. Dans cette lettre, Villers confond l'auteur de Justine, Sade, avec Choderlos de Laclos.

24 Villers 2006, 136.

25 L'identification du terme passion avec les notions des «vapeurs» et l'adjectif «hystérique» apparaît dans le Dictionnaire de l'Académie française 1798,243-4, le Dictionnaire des sciences médicales 1818, 226 et celui de Clerc 1885, 1227. 


\section{L'Amour n'est pas un fluide}

Joseph Philippe François Deleuze (1753-1835), infatigable défenseur du magnétisme animal, observait à propos du Magnétiseur amoureux, que c'était «un livre de métaphysique fort ingénieux et l'un des meilleurs traités que nous ayons sur le magnétisme». ${ }^{26}$ En effet, l'intention de Villers en écrivant ce roman n'était pas exclusivement littéraire. Il s'agit d'un texte comptant de nombreuses références philosophiques où sont discutées les principales thèses en faveur et contre la doctrine magnétique au prisme de la mise en scène d'une cure somnambulique. D'ailleurs, c'est l'unique roman écrit par Villers, dont l'ouvrage le plus connu est la Philosophie de Kant (1801), l'un des principaux travaux de vulgarisation sur ce philosophe en France pendant la première moitié du XIX ${ }^{\mathrm{e}}$ siècle. ${ }^{27}$

Comme plusieurs spécialistes l'ont suggéré, l'origine du Magnétiseur amoureux est probablement autobiographique. Un an avant sa publication, Villers séjourne à Saint-Julien, dans la maison de campagne du Marquis de Lézay-Marnésia, une personnalité qui jouera un rôle prépondérant dans la diffusion de la philosophie allemande dans les cercles francophones, et qu'il initia au magnétisme avec sa femme et sa jeune fille. ${ }^{28}$ Cette anecdote sert à Villers de fil conducteur pour exposer méthodiquement le résultat de ses propres recherches après que Puységur a vraisemblablement montré quelques résistances à l'initier au somnambulisme. Suite à ce conflit, Villiers aurait développé sa propre interprétation de cette thérapeutique. ${ }^{29}$

Le personnage principal du Magnétiseur amoureux est Valcourt, un jeune magnétiseur, défenseur passionné des thèses de Puységur, qui propose à Monsieur de Sainville de magnétiser sa fille, Caroline, une jeune femme de santé fragile à qui «les mauvais plaisans cherchent la cause de sa maladie dans son âge». ${ }^{30}$ Même si M. et Mme De Sainville se sont retirés à la campagne «pour soigner l'éducation de leur unique fruit de l'amour» ${ }^{31}$ - raconte Villers inspiré par les conseils de L'Emile ou de l'éducation (1762) - l'état de santé de la jeune fille ne semble pas s'améliorer. ${ }^{32}$

Pourtant, Madame de Sainville se montre réticente à la proposition de Valcourt et se comporte en gardienne de la moralité de sa fille face aux rapports qu'elle peut entretenir avec les hommes, «des ennemis déclarés de la

26 Mercier 1829, 102.

27 Wittmer 1908, 2. Voir aussi, Azouvi et Bourel 1991, 114.

28 Cette thèse est soutenue par Bégin 1939 et Azouvi 2006.

29 Bégin 1939, 412.

30 Villers 2006, 85.

31 Villers 2006, 85.

32 Rousseau, J.-J. 1762, 134. 
vertu des femmes». ${ }^{33}$ Elle va finalement céder à l'idée de la magnétiser suite au discours persuasif de Valcourt défendant cette doctrine face aux critiques émises dans les rapports de 1784 et reproduites grâce au personnage de l'abbé. Dans le roman, l'abbé devient ainsi le porte-parole du pouvoir politique de la société de l'Ancien Régime, celui qui refuse d'accepter la valeur scientifique du magnétisme car «ses effets sont chimériques», c'est-à-dire qu'ils sont le résultat «d'un charlatanisme abominable». ${ }^{34}$ Avouant un certain ressentiment à l'encontre de ce personnage, Villers décrit sa physionomie décadente comme celle d'un homme dont «l'esprit contenu par des organes épais, ne peut s'élancer au-delà de son enveloppe renforcée» et constitue la preuve qu'il «conserve une idée confuse d'avoir reçu jadis le bonnet de docteur en Sorbonne». ${ }^{35}$

Enfin, Villers met en scène un médecin, qui discute de manière raisonnée la doctrine de Valcourt, et qui reconnaît l'influence curative de l'affection morale chez l'homme. De cette façon, les principales forces sociales de la fin du XVIII ${ }^{e}$ siècle impliquées dans la controverse du magnétisme animal sont réunies et confrontées au cours de la narration, qui se déroule principalement dans le jardin anglais de Madame de Sainville, un lieu chargé d'érotisme depuis le succès de Julie ou la Nouvelle Héloïse (1775) de JeanJacques Rousseau. ${ }^{36}$ Le jardin symbolise en effet la nature en tant que «force sexuelle», le lieu de procréation où le magnétisme va se révéler être une sorte de désir animal face auquel l'homme n'est pas toujours capable d'ériger des

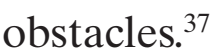

Pour Villers, comme pour Valcourt, les effets du magnétisme ne sont pas dus à la maîtrise d'un fluide mais à l'action d'une âme sur une autre exercée par la volonté du magnétiseur. ${ }^{38}$ C'est pour cela que Valcourt reconnaît qu il sera difficile de montrer les effets du magnétisme animal dans un système physique, car sa cause est immatérielle, voire spirituelle. Dans le domaine du magnétisme animal, «les expériences ne peuvent être que de pure métaphysique» - observait le jeune magnétiseur - car le magnétisme est une branche de la connaissance «à laquelle on n'est pas encore avisé

33 Villers 2006, 107.

34 Villers 2006, 87.

35 Villers 2006, 86.

36 Rousseau, J.-J. 1775, 42.

37 Lafuente et Moscoso 1999, XLIV se réfèrent au terme de «force sexuelle» quand ils discutent la nature des passions représentées dans Le magnétiseur amoureux.

38 Voir Puységur 1807,108. Cette interprétation du magnétisme révèle l'influence de la doctrine de Puységur, que ce dernier résumait dans la devise «croyez et veuillez» pour souligner que toute la base de sa science était la croyance dans sa «puissance d'actionner le principe vital» de ses semblables et «d'en faire usage». 
de toucher, et à laquelle par conséquent, il n'y a pas de termes propres adaptés». ${ }^{39}$

Aux yeux de Valcourt, le caractère révolutionnaire du magnétisme permet d'expliquer le recours aux différentes théories philosophiques comme celle des tourbillons de Descartes ou encore des modèles physiologiques et anatomiques très semblables à la phrénologie de Gall. De surcroît, il situe l'origine des sentiments dans l'organe cérébral. ${ }^{40}$

La tête est donc chez l'homme, si l'on ne se récrie pas sur l'expression, l'organe du sentiment. Le système nerveux semble être un tissu de cordes de communication, à l'aide desquelles l'âme transmet ses impressions au corps, et par lesquelles à son tour elle en reçoit. Ainsi, de la tête, l'âme transmet son action dans toutes les parties du corps, où aboutissent les nerfs, qui communiquent directement au cerveau, soit en prenant naissance de sa partie inférieure, soit de la moëlle allongée qui en est un prolongement. ${ }^{41}$

Chez Villers, les sentiments prennent la place du fluide magnétique que Mesmer décrivait dans son fameux mémoire comme un «principe agissant dans les nerfs». ${ }^{42}$ Par cette reformulation du fluide en termes de sentiments, Villers constituait sa défense du magnétisme en tenant compte des critiques des commissaires. D'une part, il refusait l'existence physique du fluide magnétique et, d'autre part, il acceptait que l'action du magnétisme soit due à l'imagination, à la force capable d'établir le rapport affectif entre l'opérateur et le patient et que Villers identifie au cours du roman avec le sentiment amoureux. ${ }^{43}$

Dans le roman, cette initiation aux idées magnétiques proposée par Valcourt est sévèrement critiquée par l'abbé, qui refusa toute valeur épistémique à cette doctrine qui, selon lui, «existe uniquement dans les têtes dérangées» ${ }^{44}$ Malgré la méfiance exprimée par l'abbé, Valcourt continue son explication des bienfaits du magnétisme pour traiter spécifiquement les maladies des nerfs, «les paralysies, les obstructions et les fièvres» qu'il attribue à des conditions propres de son temps. Ainsi, «la constitution actuelle de la société, les mœurs et les progrès des arts et surtout l'imprimerie» ont repoussé «les bornes de notre connaissance jusqu'à l'infini» et «de là les inquiétudes continuelles qui tourmentent l'âme [...] occupée sans cesse dans la faculté de penser» et qui a oublié son influence salutaire sur le corps. ${ }^{45} \mathrm{Le}$ progrès des arts, continue Valcourt en reprenant encore une fois les thèses

39 Villers 2006, 100-1.

40 Villers 1802. Dans cette lettre, il informe Georges Cuvier de la théorie de Franz Joseph Gall qu'il trouve en parfaite harmonie avec les opinions de Kant.

41 Villers 2006, 118.

42 Mesmer 1779, iii.

43 Villers 2006, 140.

44 Villers 2006, 87.

45 Villers 2006, 175.

Gesnerus 72 (2015) 
philosophiques de Rousseau, «multiplie les besoins jusqu'à l'infini», surtout chez les personnes qui s'occupent d'études sérieuses. ${ }^{46}$

Les facteurs socioculturels vont aussi jouer un rôle décisif dans l'apparition de la maladie de la jeune Caroline qui est décrite dans le récit par des symptômes comme la pâleur du visage, la tristesse ou la désillusion. Même si Valcourt ne prononce pas de diagnostic tout au long du roman, Villers nous fait comprendre grâce aux descriptions de l'état affectif de la jeune fille que la cause de sa maladie est le mariage que ses parents ont arrangé avec le Baron d'Etampes, un poison qui consomme son cœur et dont «elle ignorait les effets», et qui devient l'obstacle à la poursuite de son amour pour Valcourt. ${ }^{47}$ Précisément, c'est Caroline qui va prendre conscience de la cause de sa maladie car, tout comme les somnambules de Puységur, elle acquiert une lucidité lui permettant de reconnaître la nature de sa souffrance.

Pendant une promenade, elle demande à Valcourt s'il est possible que l'origine de sa maladie soit l'amour, qui est peut-être la cause qui entraîne «le plus cuisant des chagrins» dans le cœur des hommes. ${ }^{48}$ Le jeune magnétiseur lui répond par l'affirmative en reprenant la même rhétorique sur la maladie d'amour que celle utilisée par Caroline, et qui ne fait que renforcer le jeu de séduction entre les deux protagonistes en décrivant leur agonie comme une forme de plaisir. ${ }^{49}$

\footnotetext{
Sans doute, ma Caroline; un amant éloigné ou privé de l'objet qu'il aime, est continuellement absorbé; son âme embrasée se précipite sur sa maîtresse [...] Son existence devient à charge, il n'y tient plus que par son amour [...] Si l'amour a causé le mal, du moins l'amour a su le guérir; depuis quelques jours on n'aperçoit plus sur votre teint de signe de langueur; il est vif et coloré. ${ }^{50}$
}

Ce passage nous fait penser que Caroline souffrait de ce qui, dans la tradition médicale galénique, était connue sous le nom de «mélancolie érotique», une maladie se manifestant par une passion obsessive capable d'altérer de manière radicale le comportement du sujet qui se montre pâle, maigre, transi, sans appétit et devient, en définitive, un simple jouet de la volonté de son amant. Bien que la mélancolie ait été considérée comme une pathologie affectant plus particulièrement le sexe masculin, des médecins comme Robert Burton ou Jacques Ferrand avaient déjà attiré l'attention sur une forme particulière que prenait cette maladie chez les femmes - notamment

46 Cette idée du rapport entre la civilisation et la prolifération de maladies nerveuses avait été déjà formulée dans la médecine de Samuel-Auguste Tissot. Il existe plusieurs travaux à ce sujet dont ceux de Vila 1998 et de Barras et Louis-Courvoisier 2001.

47 Villers 2006, 95.

48 Villers 2006, 128.

49 Sur la rhétorique de la maladie d'amour, voir Dawson 2008.

50 Villers 2006, 128. 
les veuves, les sœurs, les femmes enceintes et stériles -, à cause des vapeurs vicieuses produites pendant les menstruations. ${ }^{51}$ Cette forme de mélancolie amoureuse menaçant les femmes allait être diagnostiquée au cours du XVIII ${ }^{e}$ siècle comme «hystérie», une affection résultant des vapeurs qui s'élevaient depuis la matrice de l'utérus jusqu'au cerveau à cause du réchauffement du sang. ${ }^{52}$ Ainsi, le médecin Jean-Baptiste Louyer Villermay écrivait dans son Traité des vapeurs ou maladies nerveuses: «L'hystérie dérive presque toujours de la continence, cause d'excitation. Les causes morales [...] de la névrose utérine sont presque toujours les peines du cœur, résultat d'un amour contrarié». ${ }^{53}$

Villers pense que ce genre des maladies dont les causes sont des peines morales comme le chagrin d'amour peuvent être traitées avec succès par le magnétisme animal en faisant agir l'âme sur le corps lors de la pratique du somnambulisme artificiel, qui n'est autre qu'un «sommeil naturel produit chez un homme par une surabondance d'essence spirituelle qu'un autre occasionne». ${ }^{54}$ Selon Villers, les sentiments du patient se révèlent d'une manière particulièrement nette lorsqu'il est dans cet état somnambulique, «quand les nerfs se trouvent alors saturés de ce principe du sentiment» et «sont capables d'une finesse beaucoup plus grande que dans l'état de veille». ${ }^{55}$

En cet état modifié de la conscience, la sensation la plus forte est éprouvée par le «creux de l'estomac à l'endroit qu'on nomme le plexus solaire», ${ }^{56}$ ce que Mesmer et Puységur avaient déjà identifié comme «le sixième sens», là où on pensait que tous les nerfs du corps convergeaient. ${ }^{57}$ L'estomac est

51 Sur l'influence de l'utérus dans l'apparition de la mélancolie érotique, voir Burton 1883, 250 et Ferrand 2010, 201.

52 Diderot et D'Alembert 1778,945. Dans L'Encyclopédie, la mélancolie érotique est clairement différenciée de la «fureur utérine» car cette affection «n'a pas pour objet immédiat l'acte vénérien en général, mais le désir d'y procéder avec une personne déterminée que l'on aime éperdument». Néanmoins Ferrand 2010, 77, avait considéré la fureur utérine comme une forme de mélancolie érotique. Voir aussi, Arnaud 2007,64, pour une analyse détaillée des différentes significations et connotations de «l'affection hystérique», comprise comme une espèce de mélancolie érotique par des médecins comme Joseph-Marie Joachim Vigarous.

53 Louyer Villermay 1832, 484. Cette conception de l'amour comme une maladie est alors reformulée par des aliénistes comme Jean-Etienne d'Esquirol comme deux troubles différenciés de nature mentale, «l'érotomanie» et «l'hystérie». Voir Esquirol 1815, 186-192 et Esquirol 1838, 14 et 354. Néanmoins, cette interprétation cérébrale de l'hystérie reste rare à côté de la conception dominante qui identifie l'origine de cette pathologie dans les organes génitaux, comme l'indique Arnaud 2014, 10.

54 Villers 2006, 165.

55 Villers 2006, 165.

56 Villers 2006, 173.

57 Le sixième sens devient l'organe de tous les sens à la fois dans l'état somnambulique. A ce sujet, il faut consulter Mesmer 1781,24-5 et Puységur 1813,114. 
d'ailleurs, selon Villers, le lieu organique où se forment les vapeurs qui une fois qu'elles «sont parvenues au cerveau [...] engorgent les petits vaisseaux, et gênent la circulation des humeurs», remplissant la tête d'idées étranges et extravagantes. ${ }^{58}$ En ce sens, le magnétisme peut aider «à donner du ton à l'estomac» diminuant la douleur. ${ }^{59}$

Dans ce contexte thérapeutique, les rapports moraux entre le magnétiseur et sa patiente deviennent primordiaux, car il s'agit d'établir un «transport de l'âme» qui devient possible grâce à la volonté du magnétiseur qui suit le mouvement du cœur de la patiente «en consolant ses peines» ${ }^{60}$. Cette identification de l'âme, devenue une expression habituelle dans le vocabulaire utilisé par les magnétiseurs pendant le XIX ${ }^{\mathrm{e}}$ siècle, s'établit grâce à l'imagination, qui chez Villers devient l'agent moral capable d'encourager les affections communes à la base de ce sentiment de sympathie créé entre magnétiseur et patient. ${ }^{61}$ Comme Valcourt l'explique, il n'est pas nécessaire d'invoquer un fluide dans la cure magnétique, car il suffit de l'envisager en termes de l'action qu'une âme puisse avoir sur une autre:

Lorsque je veux, cependant, faire des expériences relatives à ma manière d'envisager le magnétisme, alors je n'emploie que l'action de mon âme. Mais on conçoit que ce procédé devient plus pénible, que si l'on a recours aux sensations qui vous aident beaucoup à concentrer votre attention. Ainsi les magnétiseurs qui croient à un fluide magnétique, ayant leur imagination reposée sur ce fluide, peuvent-ils opérer de grands effets, pourvu qu'ils emploient leur imagination plutôt sur le malade que sur leur manipulation. ${ }^{62}$

La mère de Caroline, Madame de Sainville, se révèle particulièrement enthousiaste devant ce discours prononcé par Valcourt à propos de la méthode à suivre pour que la cure somnambulique ait lieu. D'ailleurs, c'est elle qui va mettre à contribution la notion de «sympathie» pour décrire les affections communes entre le magnétiseur et la patiente comme une affinité semblable au sentiment amoureux..$^{63}$

Voilà cette sympathie, ce doux rapport d'humeurs et de caractères; les voilà bien développés; ce je ne sais quoi, qui, dès la première vue, me porte vers un être plutôt que vers un autre, qui aurait cependant un extérieur plus agréable, eh bien, c'est cette rencontre de nos âmes qui étant de la même teinte, se sont bien vite liées. ${ }^{64}$

Nous sommes donc très loin de l'hypothèse originale de Mesmer sur l'existence physique d'un fluide magnétique. Chez Villers, le fluide se transforme en un échange émotionnel ainsi appelé sympathie ${ }^{65}$ représenté en termes de

58 Villers 2006, 117.

59 Villers 2006, 117.

60 Villers 2006, 117.

61 Carroy 1991, 138.

62 Villers 2006, 140.

63 Sur la signification de la sympathie, voir Dictionnaire de l'Académie française 1798, 621.

64 Villers 2006, 140.

65 Villers 2006, 140. 
rapports amoureux comme ceux entretenus entre les deux protagonistes du roman, Valcourt et Caroline. Pour Villers, les rapports magnétiques sont de la même nature que l'affection amoureuse car tous les deux sont animés par la loi de l'attraction grâce à laquelle «les jeunes individus gravitent les uns vers les autres, comme les corps célestes». ${ }^{66}$

L'amour chez Villers se révèle être à la fois une passion à l'origine de la maladie de Caroline et un sentiment qui devient la condition de possibilité de la thérapie. Cette perception ambivalente de l'amour, toujours au cœur de nos sociétés contemporaines ${ }^{67}$ nous permet d'interpréter le magnétisme non pas exclusivement comme une thérapeutique, mais aussi comme une instance de ce que Michel Foucault appelait «une connaissance érotique», c'est-à-dire, une réflexion morale sur les pratiques sexuelles qui rend visible le lien entre le savoir et le désir dans la société prérévolutionnaire française. ${ }^{68}$

\section{Le magnétisme comme connaissance érotique}

Le choix d'un titre comme Le magnétiseur amoureux n'était pas fortuit pour Villers qui mettait l'accent, d'une manière ouvertement provocatrice, sur un des aspects les plus controversés du magnétisme animal: la relation de cette doctrine avec les mours de la société française de la fin du XVIII ${ }^{\mathrm{e}}$ siècle. Comme Jacqueline Carroy l'a signalé, le magnétisme «était une forme de pratique sexuelle de la société autorisant des rapprochements inhibés, mais tout de même suggestifs» qui tolérait certains flirts tant que le comportement n'allait pas trop loin. ${ }^{69}$ D'ailleurs, cet intérêt de Villers pour la compréhension de la signification de l'amour allait inspirer en 1806 son livre intitulé L'érotique comparée, dans lequel il cherchait à comprendre les différences «entre deux conceptions différentes de l'amour» à travers une étude comparative entre la littérature allemande et française. ${ }^{70}$

Ainsi, Le magnétiseur amoureux propose une réflexion sur le magnétisme en tant que connaissance érotique, c'est-à-dire, une pratique culturelle qui permettait au sujet de se rapporter à son propre désir en définissant les limites entre la conduite normale et pathologique. Par ailleurs, Villers souligne bien que «l'attouchement n'est pas nécessaire», car la cure somnambulique «ne consiste que dans une concentration énergétique sur le malade»; il est même

66 Villers 2006, 180.

67 Schurmans 2010, 10.

68 Foucault 1994, 247.

69 Carroy 1991, 46.

70 Villers 1806.

71 Villers 2006, 162. 
déconseillé si l'on veut éviter que les jeunes gens soient tentés par «l'empire des passions» qui est si «tyrannique à cet âge». ${ }^{71}$

La manière dont je présente le magnétisme pare aux inconvénients. Le tact, ce grand séducteur, n'y est pas employé, et les détails anatomiques, desquels il faut se remplir l'imagination, ne cadrent guères avec la volupté. ${ }^{72}$

En termes de pouvoir, les rapports magnétiques se fondaient a priori sur le contrôle exercé par l'opérateur sur la patiente, ce que Villers appelait «l'ascendant». «Cette supériorité» ${ }^{73}$ sous-entend celle du masculin sur le féminin, les femmes étant considérées comme plus sensibles «aux élans de l'imagination» et par conséquent, beaucoup plus enclines aux maladies nerveuses et aux troubles de «la sensibilité sympathique de l'organisme». ${ }^{74}$ Au-delà, les rapports de pouvoir implicites dans la thérapie impliquent une réflexion sur le danger auquel sont exposées les jeunes femmes lors qu'elles se confiaient à la volonté du magnétiseur. D'autres romans de l'époque comme Pauliska ou la perversité moderne (1798) de Réveroni Saint-Cyr mettent l'accent sur ce danger quand ils représentaient la figure du magnétiseur comme un personnage sadien utilisant ses pouvoirs magnétiques pour jouir de la souffrance et de la douleur physique infligée à une jeune femme. ${ }^{75}$

Néanmoins, dans Le magnétiseur amoureux, on assiste à un renversement en termes de pouvoir car les rapports établis entre Valcourt et Caroline s'inversent. Bien que Caroline apparaisse initialement associée au rôle de la patiente, elle devient peu à peu active, une fois qu'elle prend conscience de la cause de sa maladie: l'amour «contrarié dans sa libre satisfaction». ${ }^{76} \mathrm{En}$ effet, Caroline apprend à soigner grâce au magnétisme animal et elle y parvient, guérissant sa mère de ses migraines, un mal associé aux vapeurs et qui était fréquemment utilisé en matière amoureuse comme un «prétexte pour éloigner tout importun assez hardi pour venir rompre un tête à tête». ${ }^{77}$ Caroline passe ainsi d'un rôle de sujet passif et tyrannisé par son propre désir, une aimante, à la maîtrise de sa passion à l'aide du magnétisme. De cette façon, l'infériorité de départ de la jeune femme face à Valcourt se transforme en supériorité lorsque le magnétiseur tombe amoureux d'elle et devient

72 Villers 2006, 162.

73 Villers 1996, 152.

74 Villers 2006, 121. Sur les maladies des nerfs, voir aussi Foucault 1961, 147.

75 Réveroni de Saint-Cyr 1798. Sur les connotations sexuelles des expériences imaginés sur l'électricité et le magnétisme, voir aussi Guibert 1991.

76 Martin-Lauzer 1859, 536.

77 De Paumerelle 1774,25. Concernant les traditions médicales et littéraires autour l'affiliation entre les vapeurs et la migraine comme conséquences de la vie oisive des femmes, voir Wenger $2007,175$. 
prisonnier des forces qu'il croyait contrôler. Ainsi, Villers n'hésite pas à représenter Caroline comme la gardienne de la vertu face à la démesure de la passion amoureuse qui emporte au jeune magnétiseur.

Monsieur, j'excuse votre passion. J'excuse un moment d'erreur qui, peut-être, me fait voir combien je suis aimée. Je me plais à le croire; mais réfléchissez, venez tantôt; et alors si vous avez trouvé quelque autre expédient, j’y souscrirai. ${ }^{78}$

Enfin, nous pouvons déceler la dialectique cachée dans le titre du roman: le magnétiseur amoureux, qui est à son tour magnétisé par les charmes de la jeune Caroline et perdant le contrôle, devient l'autre pôle de la relation amoureuse, un sujet dépendant de l'objet aimé. Cette interprétation de l'érotique chez Villers laisse déjà entrevoir la différence fondamentale entre la représentation du sentiment et de la passion amoureuse dans le roman. Alors que le sentiment se définit comme la manière dont nous recevons les impressions des objets extérieurs et en même temps comme un moyen thérapeutique - la sympathie «qu'on éprouve près d'un malade», les ravages de la passion amoureuse s'identifient avec une souffrance, notamment dans le cas de certaines affections comme l'hystérie alors que «l'âme se partage entre tant de sentiments confus». ${ }^{79}$

Cette tension entre la signification du sentiment et la passion amoureuse est aussi perceptible d'un point de vue anatomique, car si Villers signale le cerveau comme le siège organique des sentiments qui se transmettent ensuite par les nerfs, il élabore une rhétorique de la passion amoureuse par l'intermédiaire de l'exaltation du cœur comme organe de la sensibilité ainsi que des rapports des humeurs, c'est-à-dire de la sympathie. ${ }^{80}$ C'est précisément sur cet aspect que réside l'originalité du Magnétiseur amoureux: il cristallise un moment de transition entre la conception galénique de passions de l'âme et le sujet cérébral des émotions en élaborant la défense d'une médecine de la sensibilité ayant recours en même temps aux humeurs, aux nerfs, au cœur et au cerveau. Dans ce sens, les sentiments se révèlent chez Villers une catégorie vague incluant tant des phénomènes intellectuels - les sensations et les impressions - qu'une affection morale, la sympathie, rendant possible la communication entre l'opérateur et la patiente.

Cette ambiguïté entre le sentiment et la passion amoureuse dans Le magnétiseur amoureux montre enfin la signification changeante de l'amour à la veille de la révolution, moment où l'institution du mariage allait être reformulée dans les termes d'un contrat reposant sur la liberté de choix et

78 Villers 2006, 129.

79 Villers 2006, 99.

80 Bound-Alberti 2010, 121.

Gesnerus 72 (2015) 
des sentiments partagés, et dont la principale menace était la passion amoureuse incontrôlée, car elle pouvait devenir une maladie menant à la folie ou au suicide. ${ }^{81}$ Ainsi, le dénouement final du roman, le mariage entre Valcourt et Caroline, montre le triomphe du sentiment amoureux qui méprise les rangs sociaux et les alliances familiales sur le mariage arrangé par les parents de la jeune fille avec le Baron d'Etampes, symbolisant l'effondrement des codes amoureux de l'Ancien Régime.

\section{Bibliographie}

Arnaud, Sabine M., L'invention de l'hystérie au temps des Lumières (1670-1820) (Paris 2014)

Arnaud, Sabine M., Narratives and Politics of a Diagnosis: The Construction and Circulation of Hysteria as a Medical Category, 1730-1820 (New York 2007)

Azouvi, François/Dominique Bourel, De Königsberg à Paris. La réception de Kant en France (1788-1804) (Paris 1991)

Barras, Vincent/Micheline Louis-Courvoisier (éds.), La médecine des Lumières: tout autour de Tissot (Genève 2001)

Bailly, Jean-Sylvain, Rapport des commissaires de la Société Royale de Médecine nommés par le Roi pour faire l'examen du magnétisme animal (Paris 1784)

Bailly, Jean-Sylvain, «Rapport secret adressé au roi, sur le prétendu magnétisme animal» dans: Alexandre-Jaques F. Bertrand, Du magnétisme animal en France, et des jugements qu'en ont portés les sociétés savantes: suivi de Considérations sur l'apparition de l'extase dans les traitements magnétiques, (Paris 1826) 511-516

Bégin, Emilie-Auguste, Biographie de la Moselle, ou histoire par ordre alphabétique de toutes les personnes nées dans ce département, qui se sont fait remarquer par leurs actions, leurs talents, leurs écrits, leurs vertus ou leurs crimes, Tome IV (Metz 1832)

Bound-Alberti, Fay, Matters of the Heart: History, Medicine and Emotion (Oxford 2010)

Boquet, Damien/Nagy Piroska (éds.), Le Sujet des émotions au Moyen Âge (Paris 2008)

Boquet, Damien/Nagy Piroska, «Une histoire des émotions incarnées», Médiévales 61 (2011) 5-24

Burton, Robert, The Anatomy of Melancholy. What it is, with all the kinds, causes, symptoms, prognostics and several cures of it (London 1651, repr. Philadelphia 1883)

Bruckner, Pascal/Finkielkraut Alain, Le Nouveau désordre amoureux: essai (Paris 1977)

Carroy, Jacqueline, Hypnose, suggestion et psychologie. L'invention de sujets (Paris 1991)

Clerc, Alexis, Hygiène et médecine des deux sexes suivies d'un dictionnaire d'hygiène et de médecine (Paris1885), Tome II

Dawson, Lesel, Lovesickness and Gender in Early Modern English Literature (Oxford 2008)

81 Bruckner et Finkielkraut 1979, 141. 
De Paumerelle, C. J. de B., La philosophie des vapeurs: ou lettres raisonnés d'une jolie femme sur l'usage des symptômes vaporeux (Lausanne et Paris 1774)

Diderot, Denis/Jean Le Rond D'Alembert, Encyclopédie, ou dictionnaire raisonné des sciences, des arts et des métiers (Genève 1778)

Dictionnaire de l'Académie française revu, corrigé et augmenté par l'Académie elle-

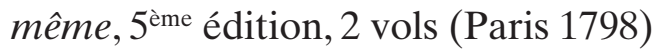

Dictionnaire des sciences médicales par une société de médecins et de chirurgiens, (Paris 1818)

Dixon, Thomas, From Passions to Emotions. The Creation of a Secular Psychological Category (Cambridge 2003)

Esquirol, Jean-Etienne-Dominique, Des maladies mentales considérées sous les rapports médical, hygiénique et médico-légal, Tome II (Paris 1838)

Esquirol, Jean-Etienne-Dominique, «Erotomanie», Dictionnaire des sciences médicales, t. XIII (Paris 1815) 186-192

Ferrand, Jacques, De la maladie d'amour, ou mélancolie érotique: Discours curieux qui enseigne à connaître l'essence, les causes, les signes, et les remèdes de ce mal fantastique (Paris 1623, repr. Paris 2010)

Foucault, Michel, Folie et déraison: Histoire de la folie à l'âge classique (Paris 1961)

Foucault, Michel, Histoire de la sexualité, vol. 2., L'usage des plaisirs (Paris 1984)

Foucault, Michel, «Un si cruel savoir» dans: Dits et écrits, vol. 1 (Paris 1994) 215-228

Guibert, Hervé, Vice (Paris 1991)

Martin-Lauzer, Auguste-Germain-Marie, Revue de thérapeutique médico-chirurgicale. Journal des connaissances médico-chirurgicales, vol. 13 (Paris 1859)

Méheust, Bértrand, Somnambulisme et Médiumnité, (Paris 1998) 2 vols.

Mercier, Alina Deldir, La vérité du magnétisme prouvée par les faits; extrait des notes et des papiers de Mme Alina d'Eldir née dans L'Hindoustan (Paris 1829)

Mesmer, Franz Anton, Mémoire sur la découverte du magnétisme animal (Paris 1779)

Mesmer, Franz Anton, Précis historique des faits relatifs au magnétisme animal jusqu'en avril 1781 (Londres 1781)

Lafontaine, Charles, Revue «Le magnétiseur: Journal du magnétisme animal», 1 $(1859 / 1860)$

Lafuente, Antonio/Javier Moscoso (éds.), Georges-Louis Leclerc conde de Buffon (1707-1788) (Madrid 1999)

Louyer Villermay, Jean-Baptiste, Traité des vapeurs ou maladies nerveuses, et particulièrement de l'hystérie et de l'hypocondrie (Paris 1832)

Pampler, Jan, «The History of Emotions: An Interview with William Reddy, Barbara Rosenwein, and Peter Stearns», History and Theory 49, 2 (2010) 247-265

Peter, Jean-Pierre, «De Mesmer à Puységur. Magnétisme animal et transe somnambulique, à l'origine des thérapies psychiques», Revue d'histoire du XIX ${ }^{\text {ème }}$ siècle, 38 (2009) 19-40

Peter, Jean-Pierre, Un somnambule désordonné?: Journal du traitement magnétique du jeune Hébert (Paris 1999)

Puységur, Armand-Marie-Jacques Chastenet de, Appel aux savants observateurs $d u$ dix-neuvième siècle, de la décision portée par leurs prédécesseurs contre le magnétisme animal, et fin du traitement de jeune Hébert (Paris 1813)

Puységur, Armand-Marie-Jacques Chastenet de, Du magnétisme animal, considéré dans ses rapports avec diverses branches de la physique générale (Paris 1807) 
Puységur, Armand-Marie-Jacques Chastenet de, Le magnétiseur amoureux (Paris 1824)

Puységur, Armand-Marie-Jacques Chastenet de, Mémoires pour servir à l'histoire et à l'établissement du magnétisme animal (Paris 1786), 2 vols

Rausky, Franklin, Mesmer ou la Révolution thérapeutique (Paris 1977)

Révéroni de Saint-Cyr, Jacques A., Pauliska ou la perversité moderne (Aurillac 1798)

Ribot, Théodule, Essai sur les passions (Paris 1907, repr. Paris 2007)

Ribot, Théodule, La logique des sentiments (Paris 1905, repr. Paris 1998)

Robaud-Luce, Maurice, Recherches médico-philosophiques sur la mélancolie (Paris 1817)

Rousseau, Georges S., «A Strange Pathology: Hysteria in the Early Modern World, 1500-1800» dans: Sander L. Gilman/Helen King/Roy Porter/Georges S. Rousseau/Elaine Showalter (éds.), Hysteria beyond Freud. (Berkeley 1993) 91-221

Rousseau, Georges S., «Nerves, spirits and fibres: Towards defining the origins of sensibility» dans: Georges S. Rousseau, Nervous acts: essays on literature, culture, and sensibility (Basingstoke 2004) 157-185

Rousseau, Jean-Jacques, Émile: ou, De l'éducation (Amsterdam 1762), Tome I

Rousseau, Jean-Jacques, Lettres de deux amants, habitants d'une petite ville au pied des Alpes, recueillies et publiées par J.J. Rousseau (Amsterdam 1775)

Schurmans, Marie-Noëlle, «D'amour et de feu», Sociologies Dossiers, Émotions et

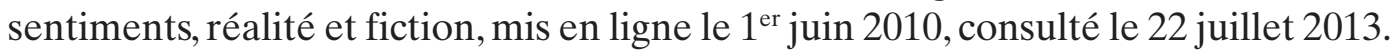
URL: http://sociologies.revues.org/3157

Starobinski, Jean, L'encre de la mélancolie, (Paris 2013)

Vidal, Fernando, «Brainhood, anthropological figure of modernity», History of the Human Sciences, 22 (2009) 5-36

Vila,Anne C., Enlightenment and Pathology: Sensibility in the Literature and Medicine of Eighteenth-Century France (Baltimore 1997)

Villers, Charles de, Lettre sur le roman intitulé Justine ou les malheurs de la vertu (Paris 1797)

Villers, Charles de, Lettre à Georges Cuvier sur une nouvelle théorie du cerveau, par le Docteur Gall; ce viscère étant considéré comme l'organe immédiat des facultés morales (Metz 1802).

Villers, Charles de, Le magnétiseur amoureux (Besançon 1787, repr. Paris 2006, édition établie par François Azouvi précédé de «La polémique du magnétisme animal» et suivi de «Documents sur l'histoire du mesmérisme»)

Villers, Charles de, L'érotique comparée (Paris 1927)

Villers, Charles de, Philosophie de Kant. Ou, Principes fondamentaux de la philosophie transcendantale (Metz 1801)

Wenger, Alexandre, La fibre littéraire: le discours médical sur la lecture au XVIII siècle (Genève 2007)

Wittmer, Louis, Charles de Villers, 1765-1815. Un intermédiaire entre la France et l'Allemagne et un précurseur de Mme De Staël (Genève/Paris 1908) 